\title{
Atomic-Layer Engineering of Oxide Superconductors
}

\author{
A. T. Bollinger ${ }^{\mathrm{a}}$, J. N. Eckstein ${ }^{\mathrm{b}}$, G. Dubuis ${ }^{\mathrm{a}, \mathrm{c}}$, D. Pavuna ${ }^{\mathrm{c}}$, and I. Božović*a \\ ${ }^{\mathrm{a}}$ Condensed Matter Physics and Materials Science Department, Brookhaven National Laboratory, Upton, NY USA \\ $11973-5000$ \\ ${ }^{b}$ Department of Physics, University of Illinois at Urbana-Champaign, Urbana, IL USA 61801-3080 \\ ${ }^{c}$ Ecole Polytechnique Fédérale de Lausanne, CH-1015 Lausanne, Switzerland.
}

\begin{abstract}
Molecular beam epitaxy technique has enabled synthesis of atomically smooth thin films, multilayers, and superlattices of cuprates and other complex oxides. Such heterostructures show high temperature superconductivity and enable novel experiments that probe the basic physics of this phenomenon. For example, it was established that high temperature superconductivity and anti-ferromagnetic phases separate on Ångström scale, while the pseudo-gap state apparently mixes with high temperature superconductivity over an anomalously large length scale (the "Giant Proximity Effect"). We review some recent experiments on such films and superlattices, including X-ray diffraction, atomic force microscopy, angle-resolved time of flight ion scattering and recoil spectroscopy, transport measurements, highresolution transmission electron microscopy, resonant X-ray scattering, low-energy muon spin resonance, and ultrafast photo-induced reflection high energy electron diffraction. The results include an unambiguous demonstration of strong coupling of in-plane charge excitations to out-of-plane lattice vibrations, a discovery of interface high temperature superconductivity that occurs in a single $\mathrm{CuO}_{2}$ plane, evidence for local pairs, and establishing tight limits on the temperature range of superconducting fluctuations.
\end{abstract}

Keywords: superconductivity, cuprates, interface, superlattice, field effect

\section{ATOMIC LAYER BY LAYER MOLECULAR BEAM EPITAXY}

Transition metal oxides have received a great deal of recent interest due to the wealth of electronic phases that can be observed in these materials, such as multiferroicity, high-temperature superconductivity (HTS), and high mobility electron gases ${ }^{1}$. The underlying cause of this variety of behaviors is the strongly correlated electrons that reside in the $d$-orbitals of these systems. At interfaces between two such oxides the competition between different states can lead to an even wider range of phenomena than is observed in the constituent materials alone ${ }^{2}$. A technical hurdle to studying these interesting oxide compounds and interfaces is that the synthesis of these chemically complex materials is typically not easily achieved. We overcome this problem by employing custom-built molecular beam epitaxy systems that allow for atomic-layer-by-layer synthesis with a high degree of control over film growth ${ }^{3-6}$.

Molecular beam epitaxy (MBE) is an ultra-high vacuum technique for deposition of thin films, typically from resistively heated thermal evaporation sources (Knudsen cells) although electron-beam sources can be used as well for refractory materials. MBE sources can be shuttered and if the system is equipped with some monitoring tools that allow for an accurate control of absolute deposition rates of respective metals, one may achieve atomic-layer-by-layer (ALL) growth. The deposition process must be controlled very accurately, at the level of one percent of an atomic monolayer or better. The BNL molecular beam epitaxy system shown in Fig. 1 is equipped with sixteen sources, a distilled ozone source, and a sixteen-channel real-time rate monitoring system based on atomic absorption spectroscopy. It is also provided with a reflection high-energy electron diffraction (RHEED) system with sample scanning capability and a timeof-flight ion scattering and recoil spectroscopy (TOF-ISARS) system for real-time chemical analysis of the film surface. These advanced surface science tools provide real-time information about the film surface morphology, chemical composition, and crystal structure, and are quintessential for the success in growing atomically smooth and perfect films. Comparable oxide MBE systems, with some variations, are in operation also at Urbana (J. Eckstein), Cornell (D. Schlom), Minnesota (A. Goldman), Pacific Northwest National Laboratory (S. Chambers), Yale (C. Ahn), Argonne (A. Bhatacharya), etc.

The controlling software allows one to control or modify the stoichiometry within each monolayer. This provides great flexibility in depositing different HTS compounds and other oxides. In particular, ALL-MBE technique is 
well suited, by design, for depositing alternating layers with the precision better than a single atomic-layer, and hence for synthesis of artificial superlattices. An important indicator is the surface roughness of such a film; in the best cases, rms roughness can be as small as 1-2 $\AA$. A typical atomic force microscope image of a $\mathrm{La}_{2-x} \mathrm{Sr}_{x} \mathrm{CuO}_{4}$ film surface gown by MBE is shown in Fig. 2a. The RHEED image recorded at the end of the film growth is presented in Fig. 2b; the intense specular spot indicates an atomically flat surface.

\section{SUPERLATTICES}

The growth technique outlined above is particularly suited to the engineering of multilayer structures. These can be specially designed to study a variety of phenomena within the high temperature superconductors. Here we will focus on those experiments that have looked at the interplay between different $\mathrm{La}_{2-x} \mathrm{Sr}_{x} \mathrm{CuO}_{4}(\mathrm{LSCO})$ and $\mathrm{La}_{2} \mathrm{CuO}_{4+\delta}$ (LCO) layers. As in all HTS materials, the phase diagram of LSCO shows the entirely distinct behaviors of insulators, superconductors, and metals depending on the doping. Therefore, many different combinations of material types can be grown using the same material family, which ensures that the interfaces between them are flawless while allowing one to explore various experimental arrangements.

One clearly important type of structure that has been studied with this technique is the superconductorinsulator-superconductor junction. A number of these were made by growing a one unit cell thick barrier layer of $\mathrm{La}_{2} \mathrm{CuO}_{4}$ between optimally doped $\mathrm{La}_{1.85} \mathrm{Sr}_{0.15} \mathrm{CuO}_{4}$ electrodes, followed by patterning and contact deposition by standard microfabrication techniques. Remarkably, when the transport along the $c$-axis was measured, no supercurrent was observed $^{7}$, even down to low temperature $(4.2 \mathrm{~K})$ and low bias $(1 \mu \mathrm{A})$ (Fig. 3a). Clearly the barrier was quite strong and free of pinholes. As a control experiment, the insulating LCO barrier could be made superconducting by annealing the structure in ozone and in this case a supercurrent could indeed be sustained at $4.2 \mathrm{~K}$. Reverting the barrier back to an insulator by annealing in vacuum was found to obstruct the flow of supercurrent once again.

The reverse geometry was also explored ${ }^{7}$ where single unit cell thick layers of $\mathrm{La}_{1.85} \mathrm{Sr}_{0.15} \mathrm{CuO}_{4}$ were grown between thicker layers of $\mathrm{La}_{2} \mathrm{CuO}_{4}$. Measurements of susceptibility showed that this superlattice was superconducting (Fig. 3b). As there are only two copper oxide planes in one unit cell of LSCO the observation of superconductivity is only possible if the very first copper oxide plane next to the insulating LCO remains superconducting. The fact that the critical temperature was not much different than what is observed in single layer LSCO films implies that the hole density in the $\mathrm{CuO}_{2}$ plane next to the LCO was not much different either. Taken as a whole these experiments show that the high temperature superconductor phase and the antiferromagnetic insulator phase do not mix and that the interface between the two must be rather sharp ${ }^{7}$.

While a proximity effect has been ruled out when the LCO layers are insulating an entirely different behavior is observed in trilayer junctions when the barrier layer is conductive ${ }^{8}$. As mentioned above, annealing an LSCO-LCOLSCO junction in ozone will turn the LCO layer superconductive due to oxygen doping while leaving the LSCO unaffected. This results in an S-S'-S type structure where the two $\mathrm{S}$ and $\mathrm{S}$ ' layers are superconducting but with $T_{c}>T_{c}$ '. The critical temperatures for $\mathrm{La}_{1.85} \mathrm{Sr}_{0.15} \mathrm{CuO}_{4}$ and $\mathrm{La}_{2} \mathrm{CuO}_{4+\delta}$ are $T_{c}=45 \mathrm{~K}$ and $T_{c}{ }^{\prime}=25 \mathrm{~K}$, respectively. For $25 \mathrm{~K}<T<45 \mathrm{~K}$ the junction then is of S-N-S type which standard theory predicts can carry a supercurrent with a magnitude that decays exponentially with the normal metal barrier thickness. Indeed a supercurrent in such S-N-S junctions was observed ${ }^{8}$ (Fig. 4a) as were clear Shapiro steps (Fig. 4b), as expected for a single Josephson junction. Note that in cuprates the coherence length for superconductivity and the mean free path are both quite short and so the normal metal coherence length, which sets the length scale for the proximity effect, is expected to be only $1-2 \AA$ (along the $c$-axis, which is the direction relevant to these experiments on devices in the small junction regime). The barrier thickness for the device shown in Fig. 4 was $100 \AA$ and according to the conventional theory no critical current should have been observed whatsoever. This is clearly not the case. Recent low-energy muon spin rotation measurements on similar structures have confirmed this picture ${ }^{9}$, showing that the barrier layer exhibits a Meissner effect (and therefore long-range phase coherence) for $T_{c}{ }^{\prime}<T<T_{c}$. This "giant proximity effect", as it has come to be known, has been demonstrated to be a real effect and one of the genuinely unique properties of the high-temperature superconductors.

\section{SIN TUNNEL JUNCTIONS}

High temperature cuprate superconductors exhibit anisotropic pairing even within the copper-oxide planes. The $d$-wave order parameter discovered by Wollman and Van Harlingen ${ }^{10}$ has regions of k-space where the pair correlations enter the condensate with opposite signs, and interference of waves from these regions can lead to weakening or even 
suppression of the order parameter. This happens at junctions and interfaces and for example leads to the formation of an Andreev bound state at surfaces where the $d$-wave order parameter is completely suppressed as first discovered by Greene and coworkers ${ }^{11}$. Even more unusual modification of the electronic structure was observed by Davidson and coworkers ${ }^{12}$ who saw broken particle-hole symmetry - indeed particle hole antisymmetry - at specular interfaces oriented such that reflections would not lead to destructive interference of the $d$-wave condensate. These unusual effects should also show up in bulk materials that have defects that lead to plane-wave reflection and scattering, such as textured films grown on coated conductors for superconducting power cables. Therefore, it is of interest to understand the origin of these changes and the degree to which they pervade imperfect samples.

Greene and coworkers discovered ${ }^{11}$ that at a (110) surface below the superconducting transition temperature a strong zero-bias conductance peak (ZBCP) develops, and they proposed that this was due to quenching of the superconducting gap because of destructive interference of order parameter components of opposite sign. Roughly speaking, near a (110) interface, the pairs incident from a direction with one order-parameter sign would reflect from the interface into a direction with the opposite sign. This exactly cancels the order parameter there and leaves behind an interface layer of normal electrons which are confined to the surface by the superconducting gap function. Thus arises an Andreev bound state.

Such signatures of quenched superconductivity arise not only at (110) interfaces, but also at a (100) interface as long as it is terminated by an amorphous insulator ${ }^{13}$. The random location of atoms at an interface to an amorphous insulator leads to non-specular reflection. This scatters 'plus-lobe' pairs into 'minus-lobe' directions. The net effect of this is to see a ZBCP very similar to that observed by Covington et al. ${ }^{11}$. Fig. 5 illustrates the orientation of $k$-space directions relative to the interface as well as the NIS tunneling spectra below $T_{c}$ that exhibit a ZBCP at low enough temperatures. In this device, the ZBCP splits below $10 \mathrm{~K}$. By $1.7 \mathrm{~K}$ the splitting is complete and a new gap feature at 1.5 $\mathrm{mV}$ emerges. This was also seen in the earlier work by Covington et al. ${ }^{11}$, and was explained as being due to a subdominant order parameter arising in the interface electron system. At low enough temperatures, superconducting order emerges with a symmetry not quenched by $k$-space scattering.

If the amorphous insulator is replaced by a crystalline one, completely different behavior is observed. This geometry is illustrated in Fig. 6a. Pictures of the RHEED patterns observed just before and just after the growth of the barrier are shown in panels $\mathrm{b}$ and $\mathrm{c}$. Atomic force microscope images of the surface flatness were obtained on similar films, and they exhibited rms roughness values less than $0.4 \mathrm{~nm}$. The films showed single steps about $0.4 \mathrm{~nm}$ tall. Thus they were suitable for making $a$-axis tunnel junction trilayers. To do this, a thin crystalline film of $\mathrm{CaTiO}_{3}(\mathrm{CTO})$ was grown on top of a flat (100) oriented layer of $\mathrm{YBa}_{2} \mathrm{Cu}_{3} \mathrm{O}_{7}$. The $\mathrm{CuO}_{2}$ planes are the corner-sharing pyramids and they terminate at the interface to CTO. A normal metal is deposited on top of the barrier, all done sequentially inside the same ultra-high vacuum MBE system.

After growth of the trilayers, the samples were processed into four-point tunnel junction devices using a selfaligned mesa structure. The device areas ranged from $30 \mu \mathrm{m}^{2}$ down to $5 \mu \mathrm{m}^{2}$. Differential conductance curves were measured from room temperature down to $4.2 \mathrm{~K}$ on more than 30 devices. A typical result is shown in Fig. 7. Just below $T_{c}$ the electrodes are all superconducting so there is no voltage drop in the on-chip wiring. All of the voltage is developed across the barrier. The curve appears roughly parabolic but with its minimum shifted away from the zero bias. This is a well known effect of barrier bending due to band line-up differences between the metal layers and the insulating barrier. Differences in the line-up between Au and CTO and between YBCO and CTO cause this effect. This makes the barrier effectively trapezoidal in shape instead of rectangular.

At $T_{c}$ a pronounced particle-hole asymmetry develops, leading eventually to a discontinuity in the tunneling conductance right at the chemical potential. For negative voltages, electrons are removed from YBCO. A gap in the density of states develops as the temperature is lowered, accompanied by a "pile-up" of spectral weight below the isosbestic point at around $20 \mathrm{mV}$. At positive voltages, extra conductance is observed uniformly. On both sides of zero bias, the temperature-dependent changes in the shape and distribution of tunneling conductance occur within $40 \mathrm{mV}$. Apparently, at $a$-axis interfaces where crystalline order is preserved, a marked breaking of particle-hole symmetry is observed. This is due to the way in which the barrier affects the HTS superconducting state.

Devices were grown with a range of barrier thicknesses. For devices with the thickest barriers the voltage drop in the wiring is a small fraction of the voltage drop across the barrier, so this allows spectroscopic tunneling to be realized above $T_{c}$. Fig. 7b shows the tunneling spectra obtained at higher temperatures. In these devices there was no sign of a pseudo-gap, contrary to what is measured by STM tunneling spectroscopy. In planar tunneling in crystalline 
samples, however, in-plane momentum must be conserved, and this causes the forward-tunneling cone to strongly focus in the forward direction, causing the tunneling to be essentially one-dimensional. In the early days of superconductivity, the spectra obtained in a planar momentum-conserving tunnel junction were discussed by Harrison and Bardeen. Harrison pointed out that in NIN tunneling no spectral information was obtained because the expression for the tunneling current is proportional to the product of the Fermi velocity and the density of states in that direction. The first is proportional to $\partial \mathrm{E} / \partial \mathrm{k}$, while the latter is proportional to $\partial \mathrm{k} / \partial \mathrm{E}$, and all spectral information is lost. However, for tunneling into and out of a superconductor, this is no longer the case, and the renormalized density of states is readily observable. Taken at the face value, then, in a junction that obeys these constraints evidence for superconductivity should be obtained as long as superconducting pairing that renormalizes the DOS is present. Since we failed to see any evidence for such pairing above $T_{c}$, we were forced to conclude that the pseudo-gapping seen in other experiments well above $T_{c}$ is not due to superconducting fluctuations. The same conclusion has been derived from direct measurements of frequency-dependent conductivity by $\mathrm{THz}$ spectroscopy ${ }^{14,15}$ and from disappearance of vortex-driven magneto-resistance oscillations in HTS nanorings ${ }^{16}$

\section{INTERFACE SUPERCONDUCTIVITY}

So far we have discussed cuprate heterostructures where at least one layer was grown from the outset such that it should be superconducting. However, when cuprate layers that are not expected to be superconducting are combined a number of interesting results have been observed as well. Bilayer films were grown utilizing insulating $\mathrm{La}_{2} \mathrm{CuO}_{4}$ (Fig. 8a) and metallic $\mathrm{La}_{1.55} \mathrm{Sr}_{0.45} \mathrm{CuO}_{4}$ (Fig. 8b) layers in both metal on insulator (I-M) and insulator on metal (M-I) configurations $^{17}$. The $R(T)$ curves of these bilayers (Fig. 8c) show high temperature superconductivity. Surprisingly, the deposition sequence matters; the critical temperature for $\mathrm{M}$-I bilayers $(\sim 30 \mathrm{~K})$ is about twice that of the I-M structures. Ozone annealing of M-I bilayers makes the upper LCO layers superconducting as expected, but the surprise here is that in such M-S' bilayers $T_{c}$ exceeds $50 \mathrm{~K}$, an enhancement of $\sim 20 \mathrm{~K}$ over the original M-I bilayer and $\sim 10 \mathrm{~K}$ higher than in optimally doped single phase LSCO films and bulk single crystals ${ }^{18}$. Such a high critical temperature was actually observed previously in similar hetero-structures ${ }^{19}$ but at that time no method was known to measure the thickness of the highest- $T_{c}$ layer. The emergence of superconductivity in the bilayers was also found to depend on the thickness of the upper layer, appearing when any number of insulating layers was added to the metallic base layer in M-I structures, whereas at least $>2$ unit cells of metallic LSCO had to be grown on the insulating base in I-M structures before superconductivity became apparent.

These straightforward observations immediately raise several questions: How does the superconductivity develop? Does the superconductivity occur right at the interface as one would presume or somewhere else within the bilayer? Why a dichotomy is observed in the critical temperatures of I-M and M-I bilayers?

To answer the first question, a superlattice (SL) was grown with each SL period containing one unit cell of $\mathrm{La}_{2} \mathrm{CuO}_{4}$ and two unit cells of $\mathrm{La}_{1.64} \mathrm{Sr}_{0.36} \mathrm{CuO}_{4}$. The SL had total of fifteen such periods. Although the compositions of the individual layers are known not to be superconducting, the SL showed ${ }^{19} T_{c}=38 \mathrm{~K}$. The structure was studied by resonant soft x-ray scattering, a technique that can differentiate between the mobile charge carriers and the lattice atoms in the sample ${ }^{20}$. In Fig. 9a we show the measured $\mathrm{Sr}$ atom distribution for one subsection of the superlattice and calculated hole distributions in the $\mathrm{CuO}_{2}$ planes. Some $\mathrm{Sr}$ diffusion into the nominal LCO layers was found to occur over a length scale of about one unit cell, in agreement with initial measurements of the strontium diffusion by TOF-ISARS ${ }^{17}$. The expected hole distribution from this strontium diffusion is also shown in Fig. 9a and it is plain that it is not enough to account for the real hole distribution. This indicates an additional electronic redistribution of charge is occurring, with charge accumulation up to 0.18 holes per copper atom on the nominally insulating side of the interface. The close-tooptimal doping on this side of the interface not only explains the origin of the interface superconductivity but also points to where it resides.

The location of the interface superconductivity was further confirmed by $\delta$-doping tomography ${ }^{21}$ in $\mathrm{M}$-I bilayers, a method that critically hinges on atomic-layer-by-layer synthesis. A small amount of copper (3\%) was replaced with zinc in predetermined $\mathrm{CuO}_{2}$ planes (Fig. 10a) as this level of doping is known to suppress the critical temperature while leaving the carrier density unchanged in both superconducting LSCO and in superconducting oxygendoped $\mathrm{La}_{2} \mathrm{CuO}_{4+\delta}$ (Fig. 10b). A series of bilayers each with the zinc $\delta$-doping occurring in a different $\mathrm{CuO}_{2}$ plane relative to the interface were grown and their superconducting transitions measured (Fig. 10c). The result is unmistakable - all films show critical temperatures of $35 \mathrm{~K} \pm 4 \mathrm{~K}$ except for those where the $\delta$-doping takes place in the second $\mathrm{CuO}_{2}$ plane on the nominally insulating side of the interface, in which case $T_{c} \approx 18 \mathrm{~K}$. This is in a good agreement with the resonant 
soft x-ray scattering study given that the metallic layer here is thicker and doped to a higher level $(x=0.45)$ compared to the previous experiment $(x=0.36)$. It is to be expected that the first $\mathrm{CuO}_{2}$ plane on the nominally insulating side of the interface should be doped higher than in that study (note that it was already slightly overdoped) and the highest critical temperature pushed out to the second $\mathrm{CuO}_{2}$ plane. Note that the $\delta$-doping study data also show that no other $\mathrm{CuO}_{2}$ plane had $T_{c}>18 \mathrm{~K}$, if any were superconducting at all.

Finally, a reason for the difference in critical temperatures for I-M and M-I bilayers was uncovered by examining the crystal structure of these arrangements by x-ray diffraction ${ }^{22}$. The $2 \theta$ scans in Fig. $11 \mathrm{a}$ show that the $c$-axis lattice constant, $c_{0}$, differs in I-M and M-I bilayers. Comparing $c_{0}$ for these structures to that for single phase I and $\mathrm{M}$ films reveals that the bilayers take on the $c_{0}$ of their bottom layers (Fig. 11b-c). This observation was accounted for by numerical simulations using an ionic crystal model, which showed that the changes in the out-of-plane lattice constant of the top layer were due to long-range Coulomb interactions between that layer and the bottom layer. The importance of the role of ionic displacements along the $c$-axis in cuprates has been recognized in other recent observations of colossal photoinduced expansion ${ }^{23,24}$. The dependence of $T_{c}$ on the $c$-axis lattice constant for I-M, M-I, and ozone annealed M-I bilayers as well as ozone annealed $\mathrm{La}_{2} \mathrm{CuO}_{4+\delta}$ single phase films was found to be linear (Fig. 11d) thus pointing to a structural explanation of the I-M and M-I bilayer critical temperature dichotomy. However, many details remain to be clarified; for example, possible selective incorporation of interstitial oxygen close to the interface. Spectroscopic methods could be used in principle to measure the oxygen content in HTS cuprates ${ }^{25}$, but the great challenge here is that this needs to be done layer-by-layer, with atomic resolution along the $c$-axis.

\section{FIELD EFFECT SUPERCONDUCTIVITY}

We have seen above that the nearly perfect interfaces made possible by the ALL-MBE technique have resulted in layers with an accumulation of mobile holes without chemical doping. This charge transfer is due to the differences in electrochemical potential between metallic and insulating cuprate layers. As such, the amount of charge that will be transferred is essentially fixed from the outset by the stoichiometry of the components of the heterostructure. An attractive alternative to this situation is to control the carriers by purely electrostatic means as is routinely done with semiconductors in field effect transistor geometries. This solid-state approach has been tried on cuprates in the past using traditional top gating, back gating, and even ferroelectric gating, but the shifts in $T_{c}$ that could be induced by field were relatively small ${ }^{26}$. A more powerful alternative is provided by electrolyte gating, as was demonstrated in a series of thorough studies begun two decades ago by McDevitt and collaborators ${ }^{27-35}$, who were able to induce shifts in critical temperatures exceeding $50 \mathrm{~K}$. Unfortunately, this large body of pioneering work was somehow overlooked by the broader HTS community (admittedly, including ourselves) and this advance had to be rediscovered.

The fabrication of a superconducting field effect transistor is beset by at least two difficult technical problems compared to semiconductors ${ }^{26}$. The first is that is that the dielectric screening length along the $c$-axis in LSCO is $(6 \pm 2) \AA$, about one-half the $c$-axis lattice constant ${ }^{20}$. Since there are two $\mathrm{CuO}_{2}$ planes in one unit cell this means that only the first $\mathrm{CuO}_{2}$ plane from the top surface of the film is significantly affected. The field becomes appreciably screened for all other $\mathrm{CuO}_{2}$ planes with a concurrent decrease in the induced mobile carriers. Perfect ultrathin films then are a necessity and here again the ALL-MBE technique provides a solution. The second difficulty is that the carrier concentration in superconducting LSCO is on the order $\sim 10^{21} \mathrm{~cm}^{-3}$. This is much higher than what is found in conventional semiconductors and requires fields of the order $10^{8} \mathrm{~V} / \mathrm{cm}$ to obtain, which is larger than the breakdown field in any known gate insulator. However, such fields can be achieved in devices using electrolyte gates to create a Helmholtz double layer at the surface of thin films ${ }^{36,37}$.

To examine the field effect on superconductivity, many ultrathin (1-2 unit cells thick) films of $\mathrm{La}_{2-x} \mathrm{Sr}_{x} \mathrm{CuO}_{4}$ were grown by ALL-MBE. The doping range of $x=0.06-0.20$ was covered. Two different electrolyte gates were applied: one was a polymer with a dissolved salt and the other was an ionic liquid. When a voltage was applied to the gate large shifts were seen in the critical temperature (up to 75-80\%) as well as in the normal-state resistance (Fig. 12a) that were reversible and reproducible. Critical temperature shifts were also observed by mutual inductance measurements as well. The field effect would work in both the enhancement mode and the depletion mode, shifting $T_{c}$ in the expected direction depending on the initial chemical doping level ${ }^{38}$.

Gate voltage scans on underdoped films allowed these samples to be tuned all the way through the superconductor insulator transition, with a separatrix between superconducting and insulating states found for $R_{\square}=R_{Q}=h / 4 e^{2}$, the quantum resistance for pairs (Fig. 12a inset). From the normal-state resistance for each $R_{\square}(T)$ curve the doping level, $x$, could be approximated and the data recast as a series of isothermal curves that shows how the 
resistance evolves with electrostatic doping at fixed temperature (Fig. 12b). All of these curves crossed at $x \approx 0.06$, in agreement with the known phase diagram of $\mathrm{La}_{2-x} \mathrm{Sr}_{x} \mathrm{CuO}_{4}$. A scaling analysis of these curves was then performed that collapsed all of the data onto a single curve (Fig. 12c), from which the critical exponent product could be extracted to be $z v=1.5$. These results are consistent with a bosonic superconductor-insulator transition in which Cooper pairs and vortices exist on both sides of the transition. In the superconducting phase the Cooper pairs are free and the vortices are localized whereas is in the insulating phase the opposite is true, the vortices freely move and the pairs are localized in a Cooper pair glass. This picture of superconductivity destroyed by quantum fluctuations at low temperature near critical doping is complementary to its destruction by thermal fluctuations ${ }^{14-16}$ at higher doping levels. Independently, very similar results were also obtained ${ }^{39}$ in $\mathrm{YBa}_{2} \mathrm{Cu}_{3} \mathrm{O}_{7-\delta}$ films (although with some details such as the estimated value of $z v$ being slightly different), so it is likely that they are universal to the HTS cuprates.

\section{CONCLUSIONS}

The precise control of growth of thin films and heterostructures by ALL-MBE has opened the door to a wide range of studies on the cuprates. The HTS phase and the antiferromagnetic insulator phase have been shown not to mix and the interface between the two found to be very sharp. The giant proximity effect has been established to be a real phenomenon, very peculiar to the HTS state. Interface superconductivity between two nonsuperconducting compounds has been demonstrated. And essentially perfect ultrathin films have been tuned electrostatically over a wide portion of the phase diagram in the vicinity of the superconducting dome. Altogether, ALL-MBE technique has proven to be a very incisive tool for the study of superconducting materials and is likely to continue playing an important role in the future.

\section{ACKNOWLEDGEMENTS}

This work was supported by the Center for Emergent Superconductivity, an Energy Frontier Research Center funded by the US Department of Energy, Office of Science, Office of Basic Energy Sciences (A.T.B., I.B., J.N.E.). D.P. and G.D. were supported by the Laboratory for Physics of Complex Matter, EPFL and the Swiss National Science Foundation.

\section{REFERENCES}

1. Takagi, H. and Hwang, H. Y., “An Emergent Change of Phase for Electronics”, Science 327, 1601-1602 (2010).

2. Mannhart, J. and Schlom, D. G., "Oxide Interfaces - An Opportunity for Electronics”, Science 327, 1607-1611 (2010).

3. Eckstein, J. N., Bozovic I. and Virshup, G. F., "Atomic Layer Engineering of High-T $\mathrm{T}_{\mathrm{c}}$ Materials and Heterostructure Devices", Materials Research Society Bulletin 19, No. 9, 44-50 (1994).

4. Bozovic, I., Eckstein, J. N. and Virshup, G. F., "Superconducting Oxide Multilayers and Superlattices: Physics, Chemistry, and Nano-engineering", Physica C 235-240, 178-181(1994).

5. Eckstein, J. N. and Bozovic, I., "High-temperature superconducting multilayers and heterostructures grown by atomic layer-by-layer molecular beam epitaxy”, Annual Reviews of Materials Science 25, 679-709 (1995).

6. Bozovic, I., "Atomic-Layer Engineering of Superconducting Oxides: Yesterday, Today, Tomorrow", IEEE Trans. Appl. Superconduct. 11, 2686-95 (2001).

7. Bozovic, I. et al., "No mixing of superconductivity and antiferromagnetism in a high-temperature superconductor", Nature 422, 873-875 (2003).

8. Bozovic, I. et al., "Giant Proximity Effect in Cuprate Superconductors", Phys. Rev. Lett. 93, 157002 (2004).

9. Morenzoni, E. et al., "The Meissner effect in a strongly underdoped cuprate above its critical temperature", Nat. Commun. 2, 272 (2011).

10. Wollman, D. A. et al., "Experimental determination of the superconducting pairing state in YBCO from the phase coherence of YBCO-Pb dc SQUIDS", Phys. Rev. Lett. 71, 2134-2137 (1993).

11. Covington, M. et al., "Observation of surface induced broken time reversal symmetry in YBCO tunnel junctions", Phys. Rev. Lett. 79, 277-280 (1977).

12. Davidson, B. A. et al., "Broken particle hole symmetry at atomically flat a-axis YBCO interfaces", Phys. Rev. Lett. 93, 107004 (2004).

13. Eckstein, J. N., et al., "Defect scattering in high $T_{c}$ and CMR tunnel junctions", Physica C 335, 184-189 (2000).

14. Corson, J. et al., "Vanishing of phase coherence in underdoped $\mathrm{Bi}_{2} \mathrm{Sr}_{2} \mathrm{CaCu}_{2} \mathrm{O}_{8+}$ ", Nature 398, 221-223 (1999). 
15. Bilbro, L. S. et al., "Temporal correlations of superconductivity above the transition temperature in $\mathrm{La}_{2-x} \mathrm{Sr}_{x} \mathrm{CuO}_{4}$ probed by terahertz spectroscopy", Nat. Phys. 7, 298-302 (2011).

16. Sochnikov, I. et al., "Large oscillations of the magneto-resistance in nano-patterned high-temperature superconducting films", Nat. Nanotech. 5, 516-519 (2010).

17. Gozar, A. et al., "High-temperature interface superconductivity between metallic and insulating copper oxides", Nature 455, 782-785 (2008).

18. Kastner, M. A. et al., "Magnetic, transport, and optical properties of monolayer copper oxides", Rev. Mod. Phys. 70, 897-928 (1998).

19. Bozovic, I. et al., "Epitaxial Strain and Superconductivity in $\mathrm{La}_{2-x} \mathrm{Sr}_{x} \mathrm{CuO}_{4}$ Thin Films", Phys. Rev. Lett. 89, 107001 (2002).

20. Smadici, S. et al., "Superconducting Transition at $38 \mathrm{~K}$ in Insulating-Overdoped $\mathrm{La}_{2} \mathrm{CuO}_{4}-\mathrm{La}_{1.64} \mathrm{Sr}_{0.36} \mathrm{CuO}_{4}$ Superlattices: Evidence for Interface Electronic Redistribution from Resonant Soft X-Ray Scattering", Phys. Rev. Lett. 102, 107004 (2009).

21. Logvenov, G., Gozar, A. and Bozovic, I., "High-Temperature Superconductivity in a Single Copper-Oxygen Plane", Science 326, 699-702 (2009).

22. Butko, V. Y. et al., "Madelung Strain in Cuprate Superconductors - A Route to Enhancement of the Critical Temperature", Adv. Mater. 21, 3644-3648 (2009).

23. Gedik, N. et al., "Nonequilibrium Phase Transitions in Cuprates Observed by Ultrafast Electron Crystallography", Science 316, 425-429 (2007).

24. Radović, Z., Božović, N. and Božović, I., "Photoinduced expansion of cuprate superconductors: Evidence of strong electron-lattice coupling”, Phys. Rev. B 77, 092508 (2008).

25. Kirrilov, D. et al., "Raman spectra of YBCO superconductors with different oxygen content", Phys. Rev. B 37 , 3360-3663 (1988).

26. Ahn, C. H. et al., "Electrostatic modification of novel materials", Rev. Mod. Phys. 78, 1185-1212 (2006).

27. Peck, S. R. et al., "Response of the double-layer capacitance of a high-temperature superconductor fluid electrolyte interface to the onset of superconductivity“, J. Am. Chem. Soc. 114, 6771-6775 (1992).

28. Haupt, S. G., Riley, D. R. and McDevitt, J. T., "Conductive polymer/high-temperature superconductor composite structures", Adv. Mater. 5, 755-758 (1993).

29. Haupt, S. G. et al., "Contact resistance measurements recorded at conductive polymer/high-temperature superconductor interfaces", J. Phys. Chem. 97, 7796-7799 (1993).

30. McDevitt, J. T., Riley, D. R. and Haupt, S. G., "Electrochemistry of high-temperature superconductors. Challenges and opportunities", Anal. Chem. 65, A535-A545 (1993).

31. Haupt, S. G. et al., "Preparation and Characterization of $\mathrm{YBa}_{2} \mathrm{Cu}_{3} \mathrm{O}_{7-\delta}-$ Polypyrrole Bilayer Structures", J. Am. Chem. Soc. 116, 9979-9986 (1994).

32. Lo, R. K. et al., "Polypyrrole Growth on $\mathrm{YBa}_{2} \mathrm{Cu}_{3} \mathrm{O}_{7-\delta}$ Modified with a Self-Assembled Monolayer of N-(3aminopropyl)pyrrole: Hardwiring the "Electroactive Hot Spots" on a Superconductor Electrode", J. Am. Chem. Soc. 118, 11295-11296 (1996).

33. Clevenger, M. B. et al., "Organic conductor/high- $\mathrm{T}_{\mathrm{c}}$ superconductor bilayer structures", Proc. SPIE 2697, 508-519 (1996).

34. McDevitt, J. T. et al., "Conductive polymer/high-T $\mathrm{T}_{\mathrm{c}}$ superconductor bilayer structures", Synth. Metals 85, 13191322 (1997).

35. McDevitt, J. T. et al., "Molecular engineering of organic conductor / high- $\mathrm{T}_{\mathrm{c}}$ superconductor assemblies", Synth. Metals 84, 407-408 (1997).

36. Ueno, K. et al., "Electric-field-induced superconductivity in an insulator", Nature Mater. 7, 855-858 (2008).

37. Ye, J. T. et al., "Liquid-gated interface superconductivity on an atomically flat film", Nature Mater. 9, 125-128 (2010).

38. Bollinger, A. T. et al., "Superconductor-insulator transition in $\mathrm{La}_{2-x} \mathrm{Sr}_{x} \mathrm{CuO}_{4}$ at the pair quantum resistance", Nature 472, 458-460 (2011).

39. Leng, X. et al., "Electrostatic Control of the Evolution from a Superconducting Phase to an Insulating Phase in Ultrathin $\mathrm{YBa}_{2} \mathrm{Cu}_{3} \mathrm{O}_{7-x}$ Films", Phys. Rev. Lett. 107, 027001 (2011). 


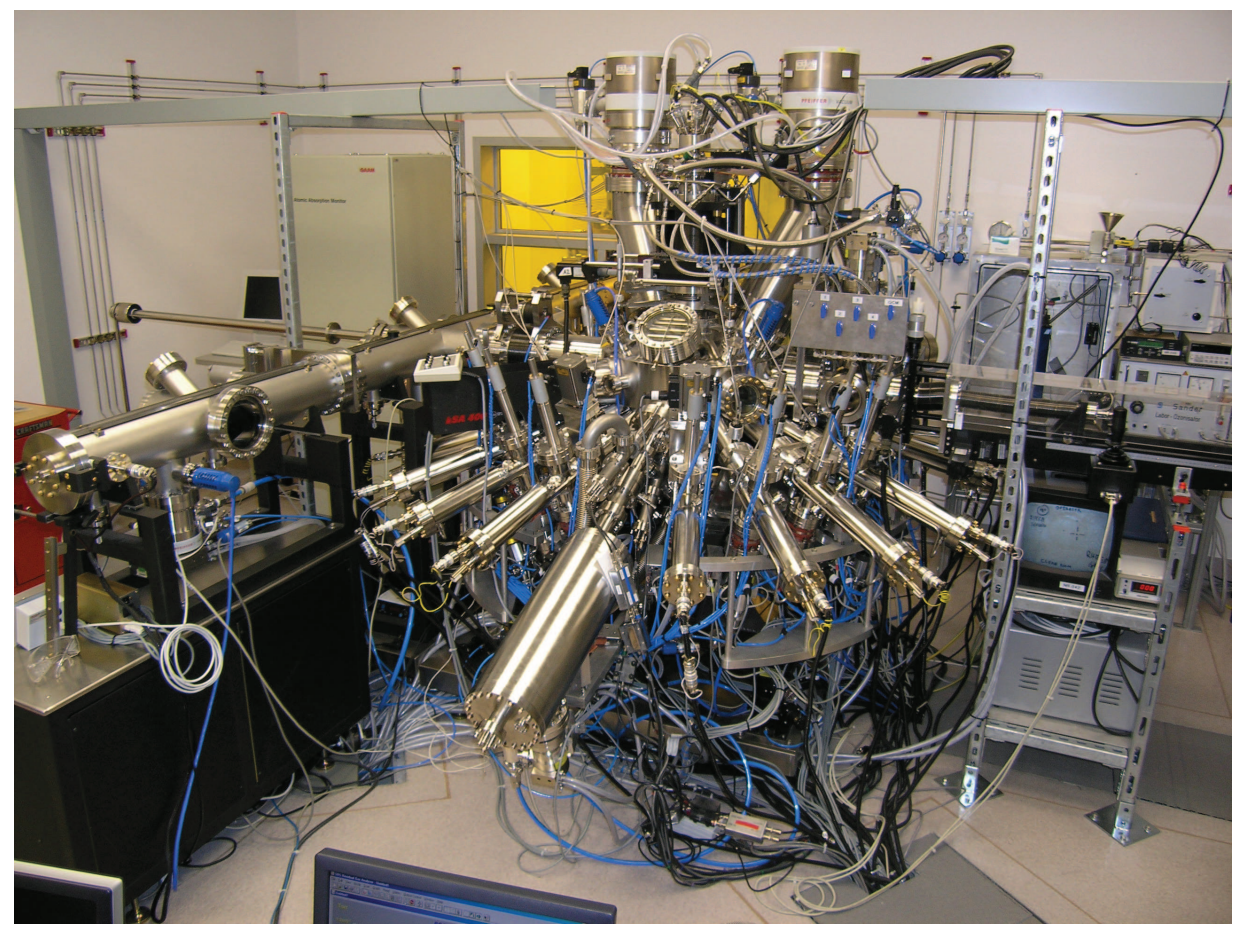

Figure 1. The atomic-layer-by-layer molecular beam epitaxy (ALL-MBE) system at BNL. 

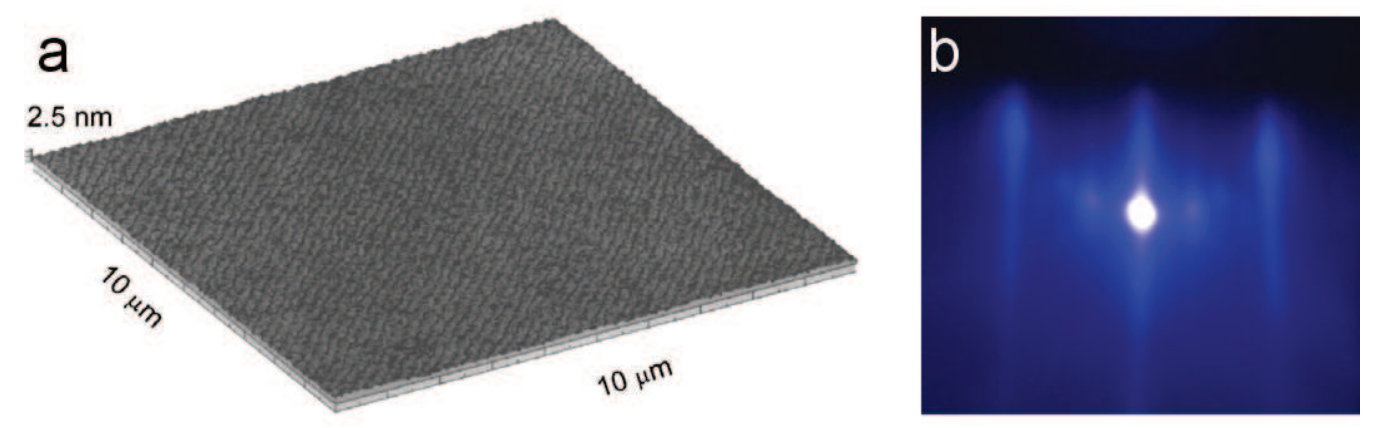

Figure 2. (a) Atomic force microscopy image of a $78 \mathrm{~nm}$ thick $\mathrm{LSCO}$ film on $\mathrm{LaSrAlO}_{4}$ substrate. It shows terraces, about $300 \mathrm{~nm}$ wide, between one-unit-cell-tall steps as a consequence of a slight $\left(0.1^{\circ}\right)$ miscut of the substrate. The rms surface roughness of this film was $3 \AA$ over an area of $2,500 \mu \mathrm{m}^{2}$. (b) Reflection high energy electron diffraction (RHEED) image taken at the end of LSCO film growth. 

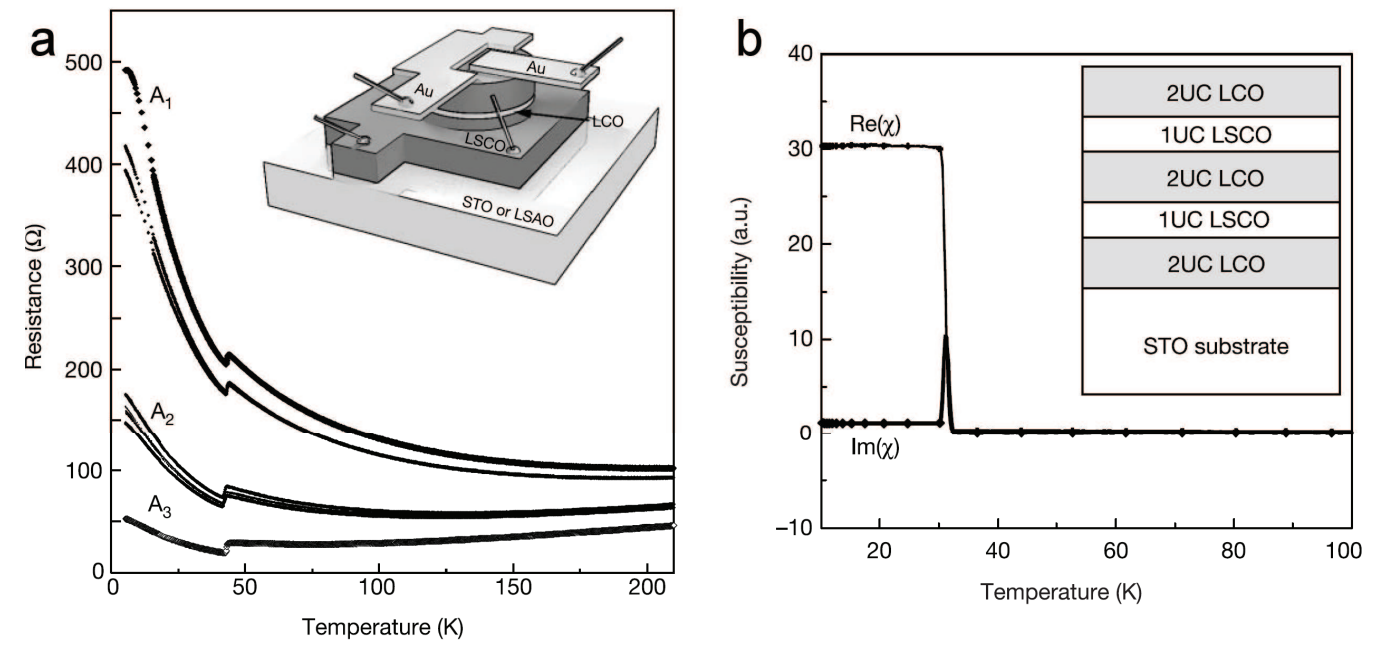

Figure 3. (a) Resistance as a function of temperature for S-I-S junctions ${ }^{7}$, where $\mathrm{S}=\mathrm{La}_{1.85} \mathrm{Sr}_{0.15} \mathrm{CuO}_{4}$ (LSCO) and $\mathrm{I}=\mathrm{La}_{2} \mathrm{CuO}_{4}$ (LCO). The mesa cross-section areas are $A_{1}=180 \mu \mathrm{m}^{2}, A_{2}=310 \mu \mathrm{m}^{2}$, and $A_{3}=710 \mu \mathrm{m}^{2}$. The dip at $T=42 \mathrm{~K}$ is the critical temperature of the LSCO bottom and top electrodes. (inset) S-I-S device structure. One unit cell barrier of insulating LCO is sandwiched between two optimally doped LSCO electrodes. The substrates used were either $\mathrm{LaSrAlO}_{4}$ (LSAO) or $\mathrm{SrTiO}_{3}$ (STO). (b) Susceptibility of LSCO-LCO superlattice. (inset) Superlattice device structure. The actual superlattice was comprised of 20 periods, each containing a two-unitcells-thick layer of insulating LCO and one-unit-cell-thick layer of optimally doped LSCO. 

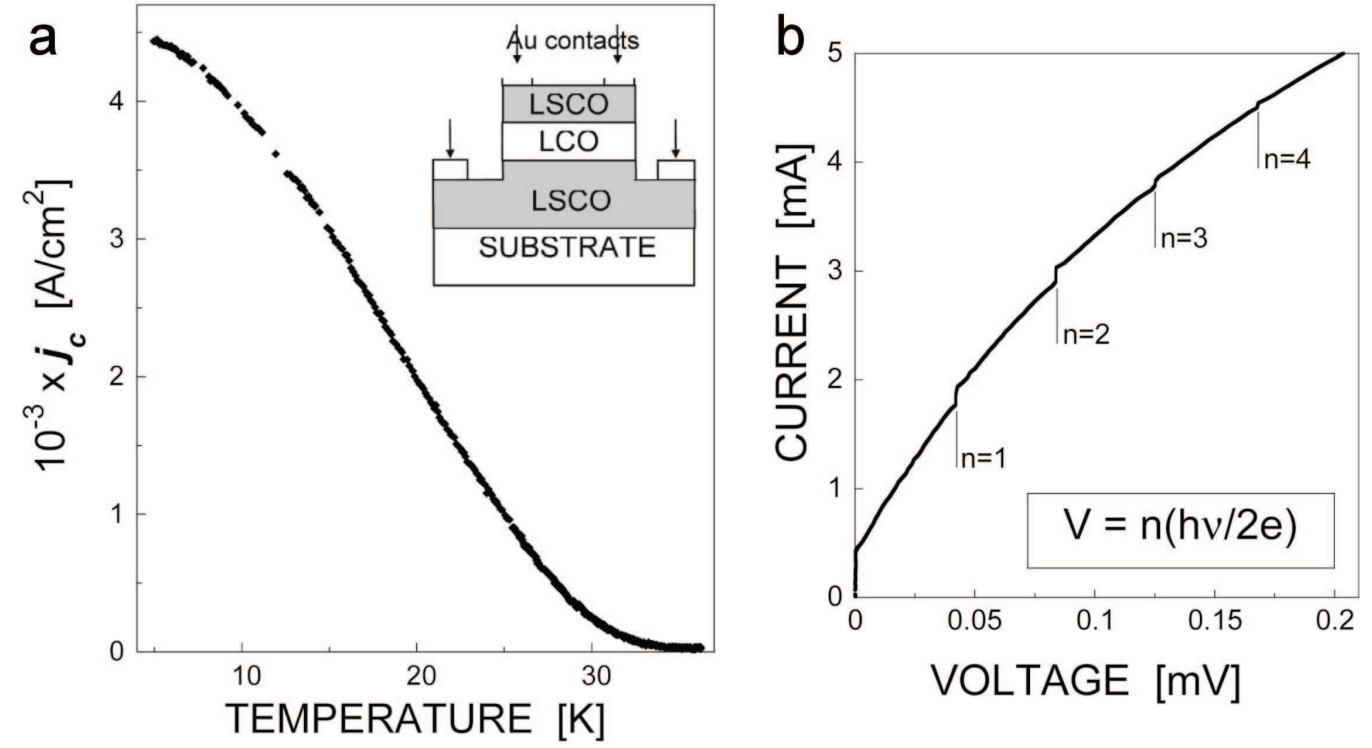

Figure 4. (a) The critical current density as a function of temperature for an $\mathrm{S}-\mathrm{S}$ '-S trilayer junction ${ }^{8}$, where $\mathrm{S}=\mathrm{La}_{1.85} \mathrm{Sr}_{0.15} \mathrm{CuO}_{4}$ and $\mathrm{S}^{\prime}=\mathrm{La}_{1.85} \mathrm{Sr}_{0.15} \mathrm{CuO}_{4} \mathrm{~A}$ large supercurrent is observed above the critical temperature of the $\mathrm{La}_{2} \mathrm{CuO}_{4-\delta}$ layer $\left(T_{c}{ }^{\prime} \approx 25 \mathrm{~K}\right)$ even though its thickness $(100 \AA)$ is two orders of magnitude larger than the normal metal coherence length (1-2 $\AA$ ). (inset) Trilayer junction structure. Top and bottom electrodes are made of optimally doped $\mathrm{La}_{1.85} \mathrm{Sr}_{0.15} \mathrm{CuO}_{4}$ and the barrier of underdoped $\mathrm{La}_{1.85} \mathrm{Sr}_{0.15} \mathrm{CuO}_{4}$. (b) Shapiro steps observed at $T=30 \mathrm{~K}$ when the junctions was exposed to microwaves $(v=20 \mathrm{GHz})$. Clear steps are observed when the Josephson frequency is an integer multiple of the microwave frequency. 

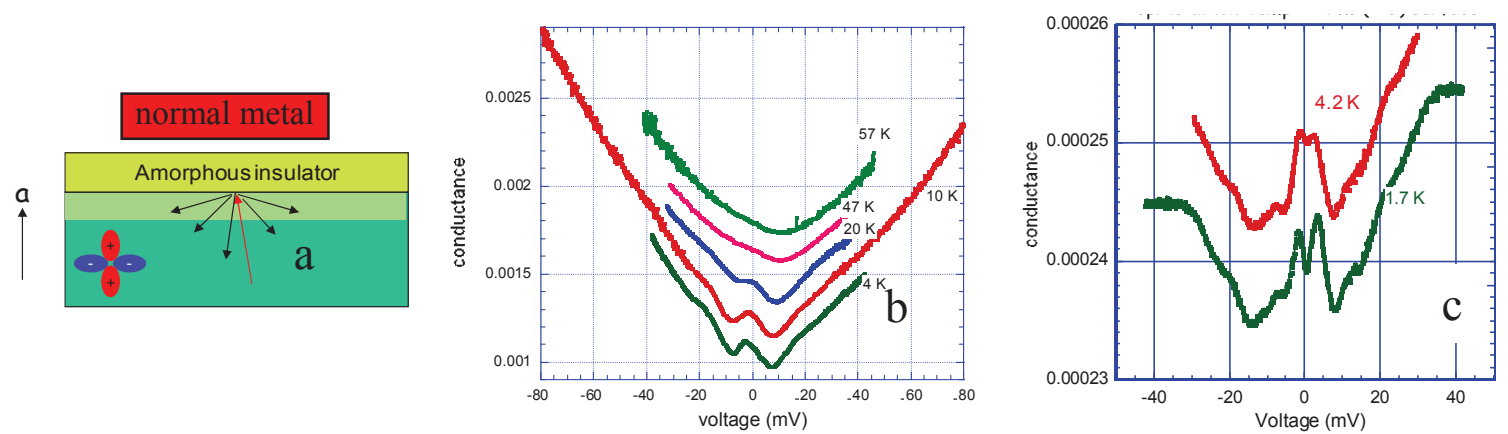

Figure 5. (a) YBCO NIS tunnel junction oriented in the a-axis direction, with an amorphous insulating tunnel barrier $^{12,13}$. Cancellation of order-parameter wave function is caused by non-specular reflection from barrier (black arrows) of incident electron waves (red arrow). The lighter area at the interface represents the Andreev bound state there. (b) Differential conductance versus voltage for temperatures between $4 \mathrm{~K}$ and $57 \mathrm{~K}$, showing ZBCP developing below $20 \mathrm{~K}$. (c) The same, but for a lower temperature in a different sample. The ZBCP splits below $10 \mathrm{~K}$, providing evidence that electrons at interface fall into a sub-dominant order parameter at low enough temperatures, similar to what Covington et al. ${ }^{11}$ reported in (110) NIS tunnel junctions. 


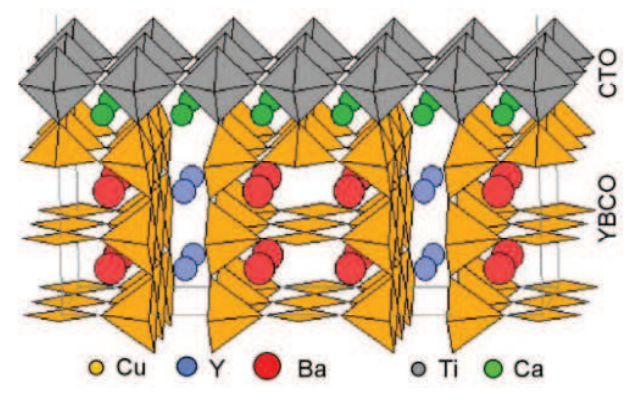

a
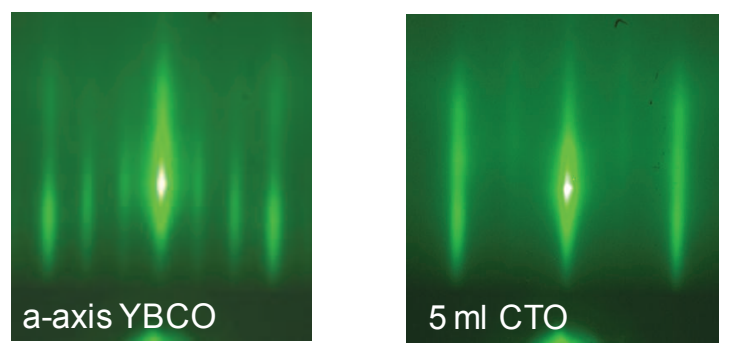

b

C

Figure 6. (a) The schematic structure of the $a$-axis YBCO-CTO interface ${ }^{12,13}$. (b) The RHEED pattern seen on the surface of YBCO just before the growth of the CTO barrier layer. (c) The RHEED pattern seen just after the barrier layer (less than $2.0 \mathrm{~nm}$ thick) was completed. 

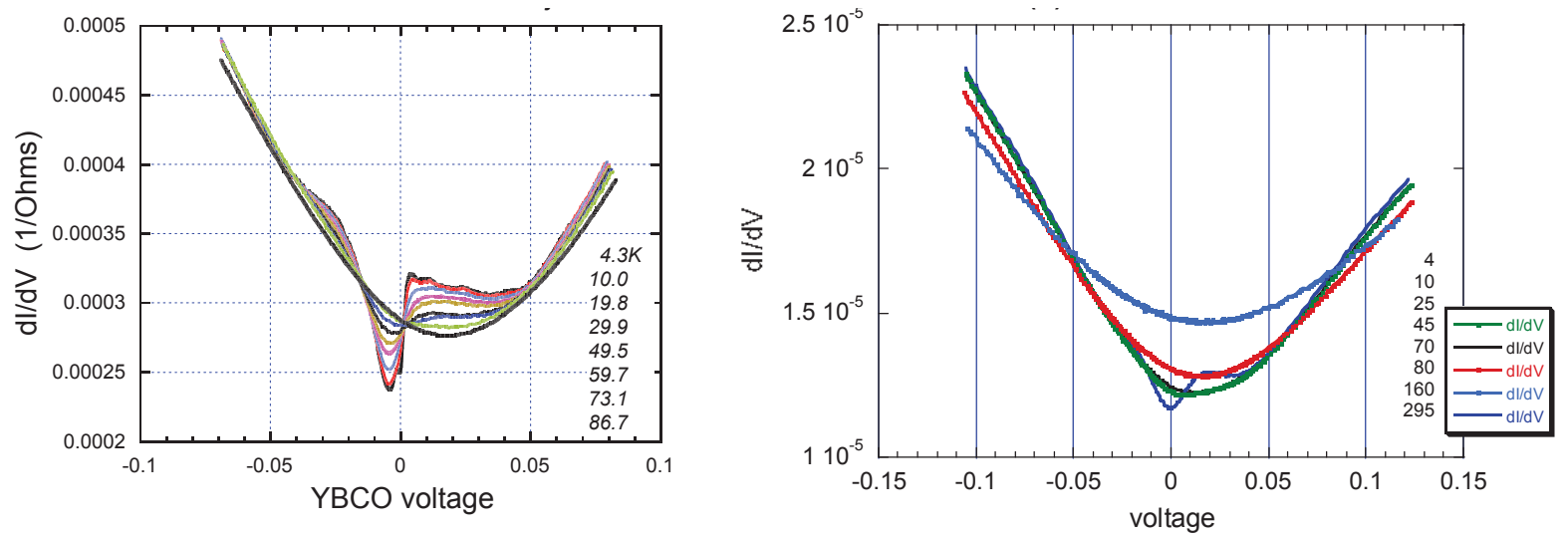

Figure 7. (a) Differential conductance spectra ${ }^{12,13}$ taken at various temperatures between $4.3 \mathrm{~K}$ and $88 \mathrm{~K}$ (= the $T_{c}$ of the device). (b) Differential conductance spectra taken above $T_{c}$ in another device with a less transparent barrier. 

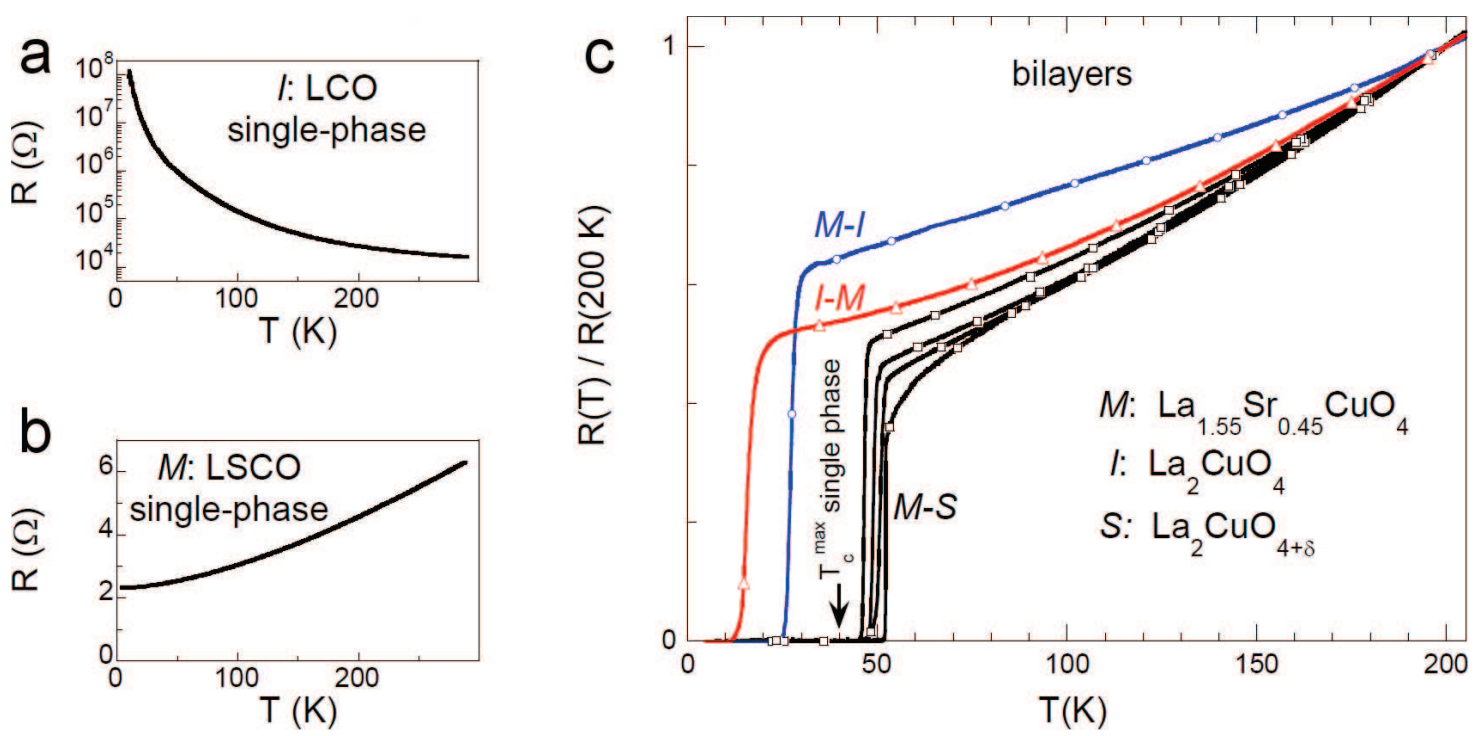

Figure 8. (a) Single phase LCO film $R(T)$ displays insulating behavior. (b) Single phase LSCO film $R(T)$ is metallic. (c) Bilayer structures composed of insulating LCO and metallic LSCO are superconducting with critical temperatures that depend on the growth sequence and that are enhanced by ozone annealing ${ }^{17}$. 


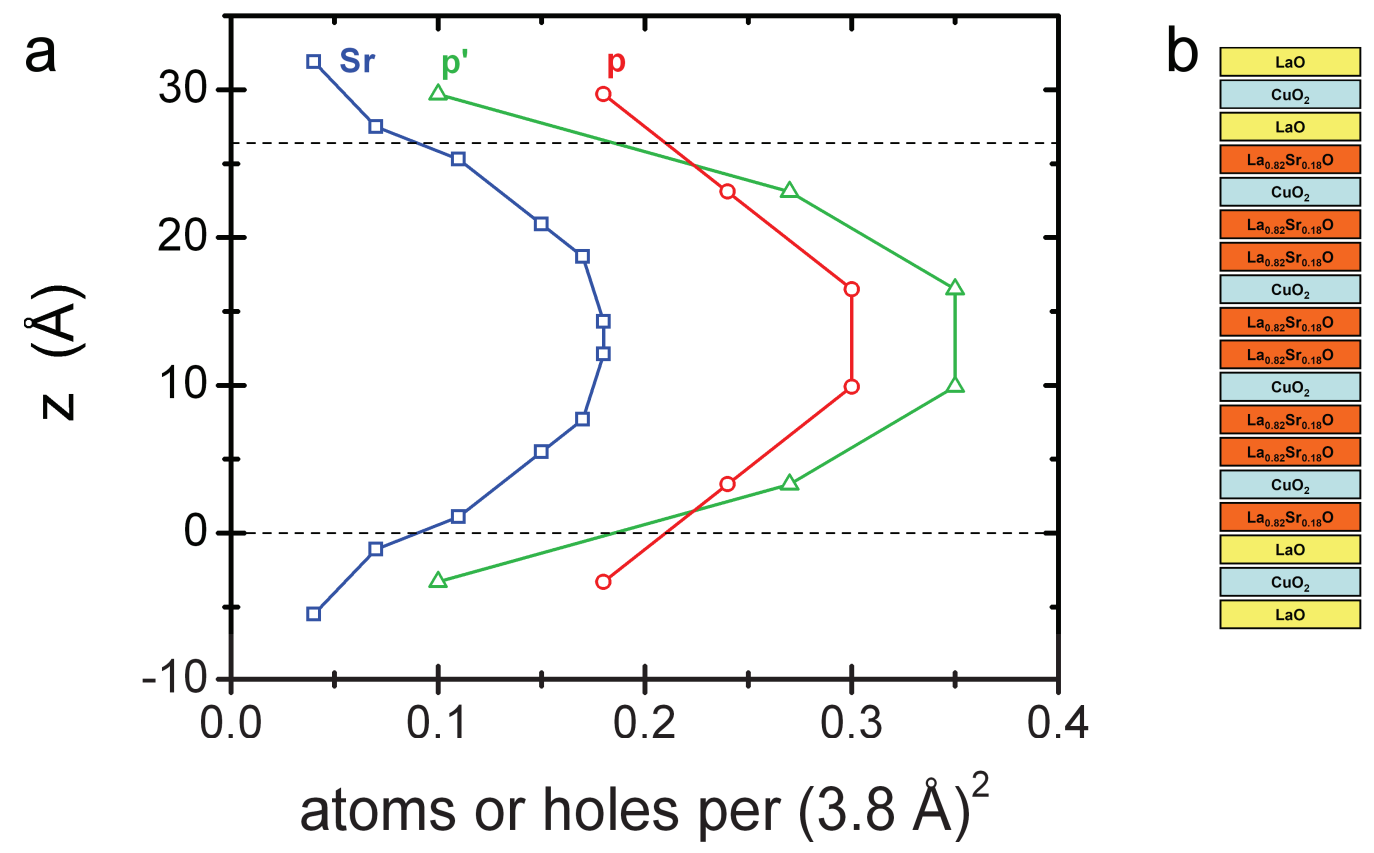

Figure 9. (a) Measured profiles of strontium atoms ( $\mathrm{Sr}, \square)$ and mobile holes $(\mathrm{p}, \circ)$ in a LCO-LSCO superlattice structure $^{20}$. The mobile hole density is clearly higher in the LCO layers than what is expected if the doping were due solely to cation diffusion (p', $\Delta$ ). Thus an electronic redistribution of charge is occurring in these structures, with depletion in the LSCO and accumulation in the LCO. (b) Schematics of the superlattice structure studied here; the layers are aligned with the z-axis of panel (a). 

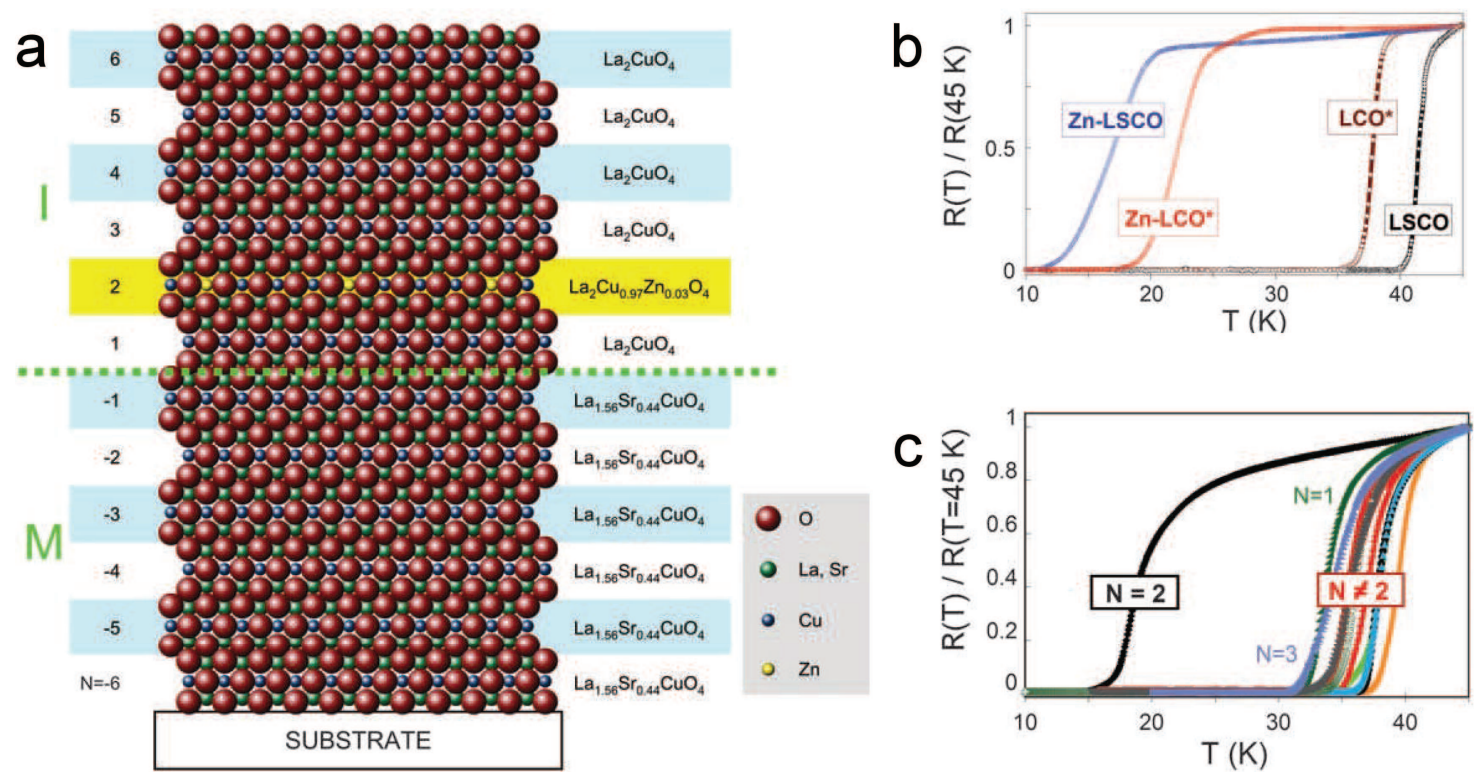

Figure 10. (a) Schematic of the $\delta$-doping experiment ${ }^{21}$. A series of M-I bilayer samples was grown, each with a single layer that had 3\% of its $\mathrm{Cu}$ atoms replaced with $\mathrm{Zn}$. (b) Comparison of resistance measurements taken on single phase optimally doped LSCO with zinc substitution (Zn-LSCO) and without (LSCO) shows that the critical temperature is greatly reduced by the Zn. Oxygen doped LCO films with and without zinc doping (Zn-LCO and $\mathrm{Zn}$-LCO*) displays the same behavior. (c) Only the bilayer film in which $\mathrm{Zn}$ was added to the second layer on the insulating side of the interface showed a marked reduction in $T_{c}$, proving that the high temperature interface superconductivity occurs only in that particular copper oxygen plane. 

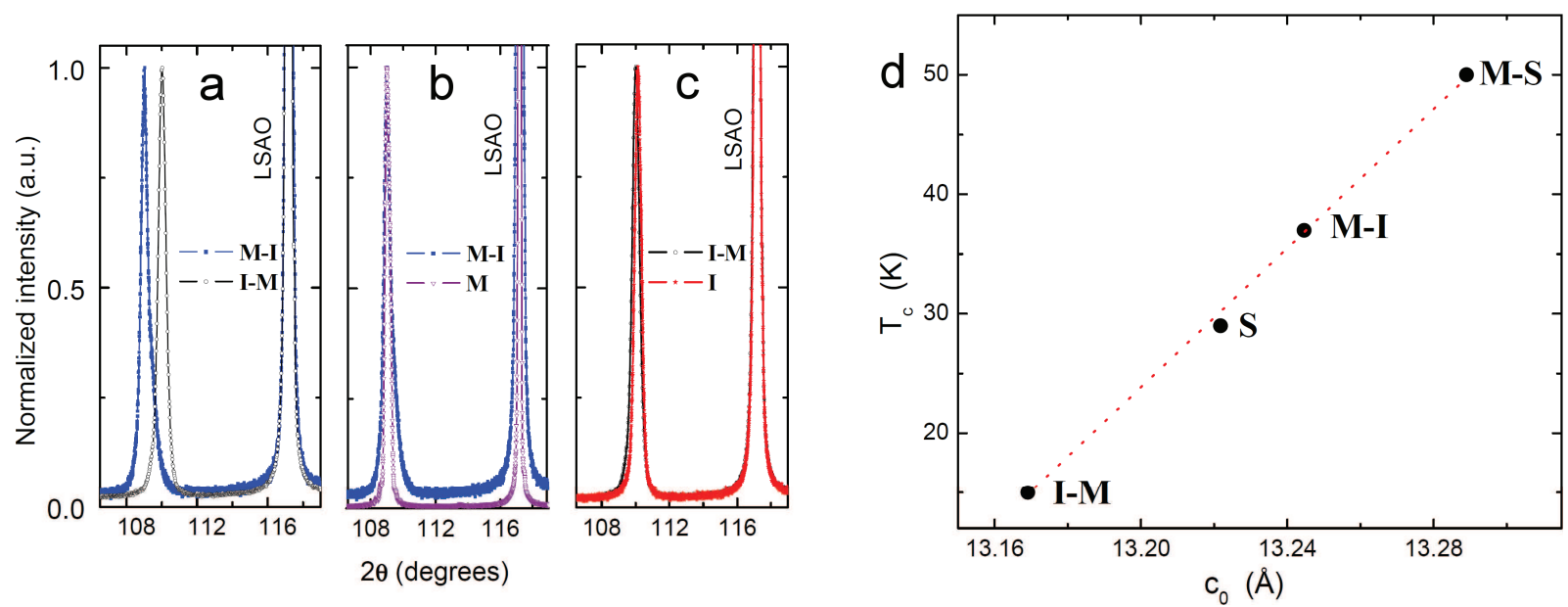

Figure 11. (a) Comparison of the x-ray diffraction of M-I and I-M bilayers reveals that these structures have only a single peak and that the two structures have different lattice constants ${ }^{22}$. (b) An entire M-I bilayer takes on the same lattice constant as the base M layer. (c) I-M bilayers show the same behavior, with the whole bilayer taking on the lattice constant of the base I layer. (d) The critical temperature is found to vary approximately linearly with the $c$-axis lattice constant. 

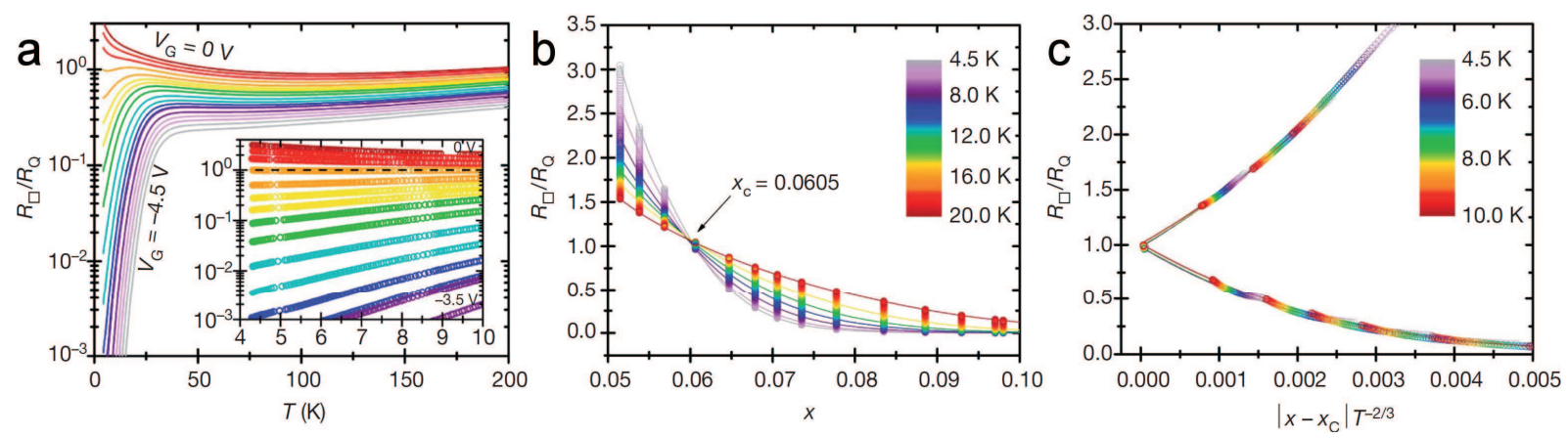

Figure 12. (a) As gate voltage is applied from $0 \mathrm{~V}$ to $-4.5 \mathrm{~V}$ an underdoped LSCO film is tuned ${ }^{38}$ from insulating to superconducting through a quantum phase transition, with a separatrix (inset) at $R_{\square}=R_{Q}$. (b) The inverse representation of the data. Each vertical set of data points corresponds to one $R(T)$ curve in (a), i.e. to one fixed carrier density. (c) This set of data collapses to a single curve by scaling the abscissa by $\left|x-x_{c}\right| T^{-1 / z v}$, where the critical exponent is found to be $z v=1.5$. 